\title{
Tidal triggering of earthquakes in Japan related to the regional tectonic stress
}

\author{
Sachiko Tanaka*, Masakazu Ohtake, and Haruo Sato \\ Department of Geophysics, Graduate School of Science, Tohoku University, Sendai 980-8578, Japan
}

(Received September 5, 2003; Revised April 22, 2004; Accepted April 26, 2004)

\begin{abstract}
We observe a correlation between the Earth tide and earthquake occurrence that is closely related to the regional tectonic stress. We investigate the direction of the tidal compressional stress using shallow earthquakes occurring in 100 subregions of Japan for nearly five years. The azimuthal distribution of the compressional stress obtained for the observed earthquake data is compared with that synthesized for random earthquake occurrence. Statistical analysis confirms a significant difference between the observed and random catalogs for 13 subregions, which include the areas where unusual seismic activities took place recently, and where the possibility of future large earthquakes has been argued. For these subregions, earthquakes preferentially occur when the tidal compressional stress is near the dominant direction of $\mathrm{P}$-axes of focal mechanisms obtained in the corresponding subregions. This suggests that the tidal stress may encourage earthquake occurrence when it acts in the direction to increase the regional tectonic stress.
\end{abstract}

Key words: Tidal triggering of earthquakes, tectonic stress, statistical test.

\section{Introduction}

The tidal force produces periodic stress variations of the order of $10^{3} \mathrm{~Pa}$ in the Earth. This stress change is much smaller than the typical stress drop of earthquakes, but its rate is generally larger than that of tectonic stress accumulation. Therefore the tidal stress could trigger an earthquake when the stress in the focal region is close to the critical level to release an earthquake. Accordingly, many studies have investigated the correlation between the Earth tide and earthquake occurrence (e.g., Emter, 1997; Glasby and Kasahara, 2001).

In order to physically investigate the relation between the Earth tide and fault rupture, recent studies have examined stress components resolved on the fault plane by using the focal mechanism solution (Tsuruoka et al., 1995; Vidale et al., 1998; Wilcock, 2001; Tanaka et al., 2002a, b). Some of these studies have also examined the trace of the stress tensor, which is invariant with coordinate rotation and corresponds to the confining stress (Tsuruoka et al., 1995; Wilcock, 2001; Tanaka et al., 2002b). Through the analysis of this component, Tanaka et al. (2002b) suggested that earthquake occurrence is encouraged when the tidal stress acts in the same sense as the tectonic stress. They analyzed earthquakes of $M_{w} \geq 5.5$ that had focal mechanisms in the Harvard catalog.

In this paper, we further explore the relation between the tidal effect on earthquake occurrence and the tectonic stress field by using a large number of shallow earthquakes occurring in Japan. These earthquakes include small ones for which focal mechanisms are not available, so we instead fo-

\footnotetext{
*Present address: National Research Institute for Earth Science and Disaster Prevention, Tsukuba 305-0006, Japan.

Copy right(c) The Society of Geomagnetism and Earth, Planetary and Space Sciences (SGEPSS); The Seismological Society of Japan; The Volcanological Society of Japan; The Geodetic Society of Japan; The Japanese Society for Planetary Sciences; TERRAPUB.
}

cus on the direction of the tidal compressional stress to assess the possible tidal effect.

\section{Data}

From the earthquake catalog of the Japan Meteorological Agency (JMA) for the period from October 1997 to May 2002, we use the origin times and hypocenters of shallow earthquakes (focal depth $\leq 70 \mathrm{~km}, M_{\mathrm{JMA}} \geq 2.0$ ) occurring in Japan. Clustered events are removed following the method of Reasenberg (1985). Dividing the whole of Japan into $1^{\circ} \times 1^{\circ}$ subregions, we select 100 subregions for the analysis, each of which includes at least 200 earthquakes. These subregions are indicated by small squares in Fig. 1. A total of 89,504 earthquakes is used in this study.

\section{Method of Statistical Test}

We investigate the correlation between the Earth tide and earthquake occurrence by statistically comparing the azimuthal distribution of a tidal stress component obtained for the observed earthquake occurrence with that synthesized for random earthquake occurrence. The random occurrence data are generated by randomly assigning 100 origin times for each hypocenter.

For the tidal azimuth, we use the horizontal projection of the maximum principal axis (maximum compression) of the tidal stress tensor considering that the horizontal compression is dominant in the Japan region. The tidal stress tensor is theoretically calculated at the origin time and the hypocenter of each earthquake for the Preliminary Reference Earth Model (Dziewonski and Anderson, 1981) following the method of Tanaka et al. (2002b). This calculation includes both the direct solid Earth tide and indirect term due to the ocean loading by using the ocean tide model NAO.99b (Matsumoto et al., 2000; Takanezawa et al., 2001). From the tidal stress tensor thus calculated, we obtain the azimuth of 


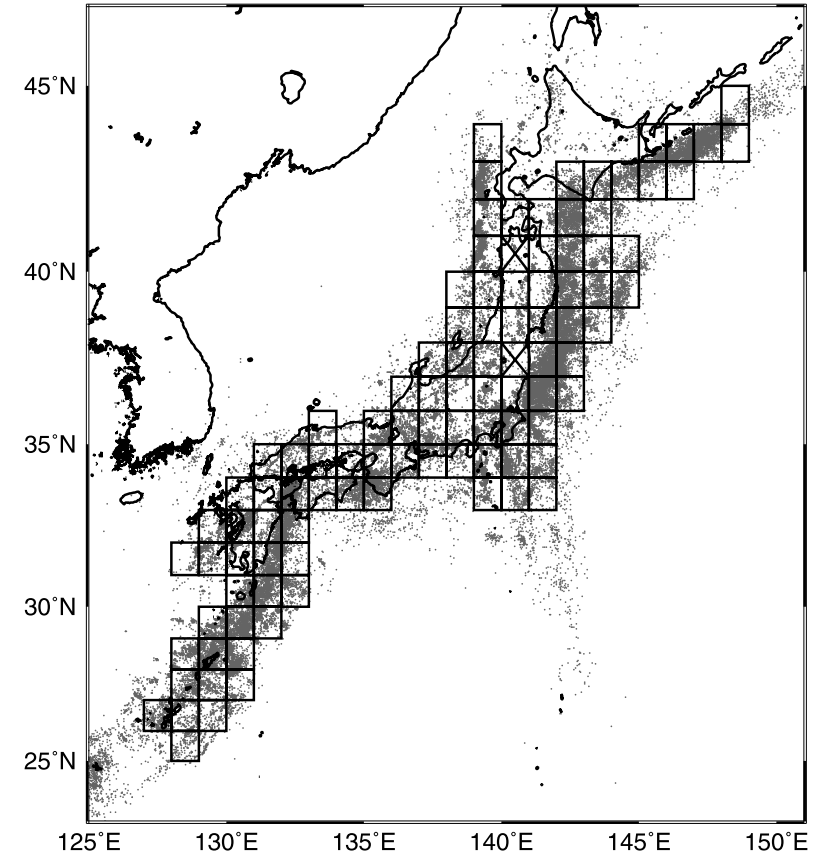

Fig. 1. Epicentral distribution of shallow earthquakes (focal depth $\leq 70 \mathrm{~km}$, $M_{\text {JMA }} \geq 2.0$ ) listed in the catalog of the Japan Meteorological Agency (JMA) for the period from October 1997 to May 2002. Small squares indicate the 100 subregions analyzed in this study.

the maximum compression for each of the observed earthquake data. The azimuth of the tidal compressional stress is also calculated for the synthesized random data. For each subregion, we obtain two frequency distributions of the azimuthal angles; the distribution for the observed earthquake data and that for the synthesized random data.

We statistically compare these two distributions by using the $\chi^{2}$ test. In this test, we assess the significance level $p$ for rejection of the null hypothesis that these two azimuthal distributions are samples from the same population. A significant difference between the two distributions means that earthquakes do not occur randomly with the azimuth of the tidal compressional stress, so that smaller $p$-value indicates a higher correlation between the Earth tide and earthquake occurrence.

\section{Result}

Figure 2 shows the result of the $\chi^{2}$ test (Fig. 2(a)) together with the number of the earthquakes, $N$ (Fig. 2(b)), for each subregion. Darker shades represent smaller $p$-values and larger numbers of the earthquakes. We find insignificant correlation between the Earth tide and earthquake occurrence for most of the 100 subregions we examined. However, small $p$-values are obtained for some subregions: $p<5 \%$ for one subregion and $5 \% \leq p<10 \%$ for twelve subregions. A systematic correlation is not seen between the observed $p$-value and the number of the earthquakes. The tidally-correlated subregions are associated with a variety of tectonic environments. Seven of them correspond to the subduction zone, four to the intraplate region with active faults, and two to the volcanic area. The smallest $p$-value of $2.0 \%$ is observed for the Miyake Island subregion as known for a typical volcanic area. We have also analyzed subregions shifted by half of the (a)

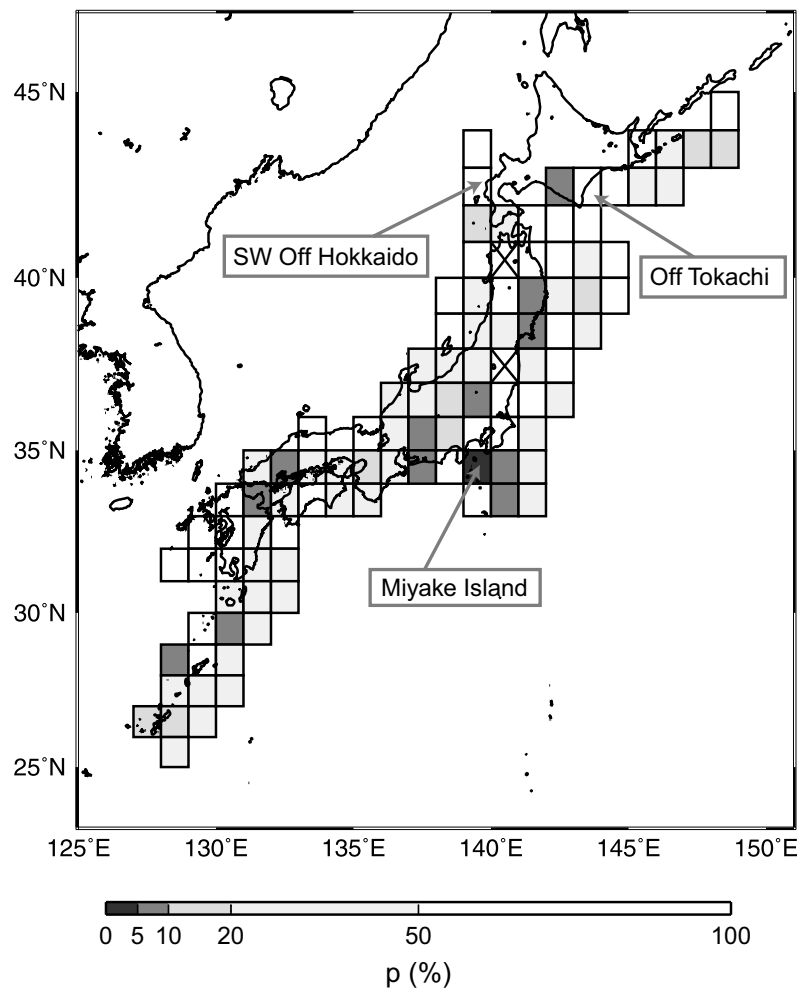

(b)

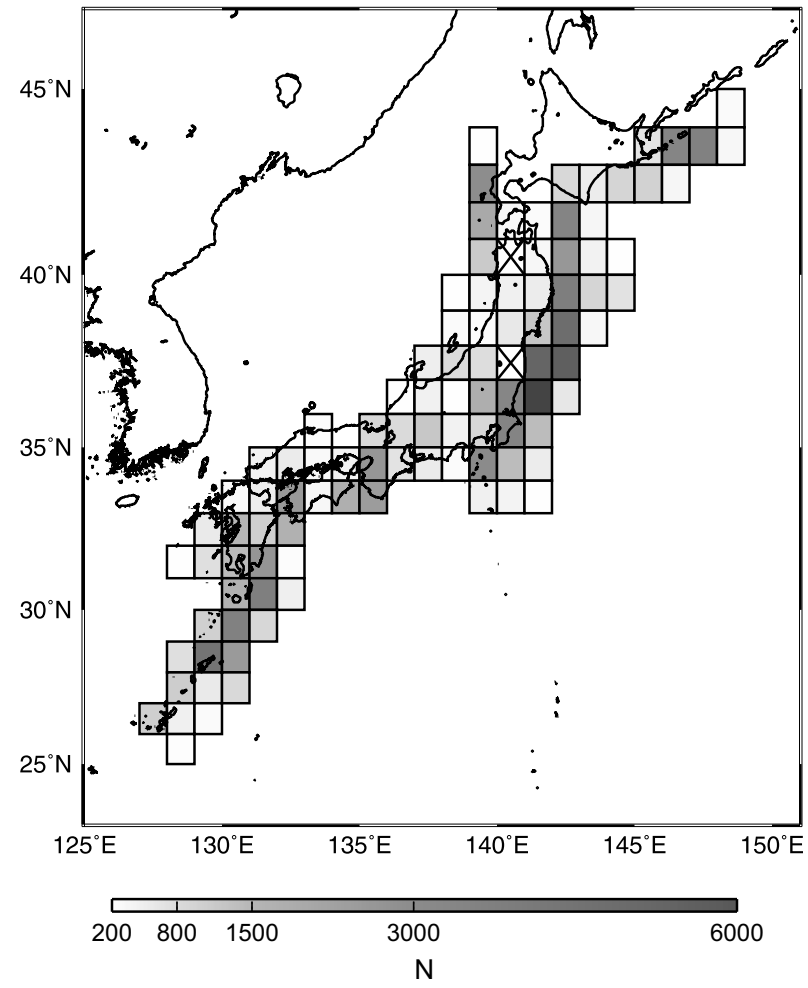

Fig. 2. Result of the $\chi^{2}$ test (a) and the number of the earthquakes (b) for each subregion by the gray scales. The scales are shown at the bottom. Darker shades represent smaller $p$-values and larger numbers of the earthquakes. 
grid size, and confirm that the same result is obtained.

Figure 3 shows examples of the azimuthal distributions of the tidal compressional stress for the observed earthquake occurrence compared with random occurrence. Note that the azimuth of the tidal compressional stress does not distribute uniformly even for the synthesized random occurrence as shown in Figs. 3(b) and 3(e). This preference of the azimuth arises by two reasons; the orbit of the Moon and the Sun on the celestial sphere lies within a limited zone for the direct term of the Earth tide, and the azimuthal distribution of the ocean loading sources is fixed when an observation point is fixed for the indirect term. In and around the Japan region, the azimuth tends to concentrate near the north-south direction. Figures 3(a) and 3(b) are the distributions obtained for the Southwestern Off Hokkaido subregion with $p$-value as large as $94 \%$ (of which location is marked in Fig. 2(a)), while Figures 3(d) and 3(e) are for the Miyake Island subregion with the smallest $p$-value of $2.0 \%$. For the case of the Southwestern Off Hokkaido subregion, we see little difference between the two distributions, which is also confirmed by a large $p$-value. For the Miyake Island subregion also, a remarkable difference is not identified visually between the two distributions although the statistical test detected a significant difference between them.

In order to make visible the difference between the two

(a) Observed

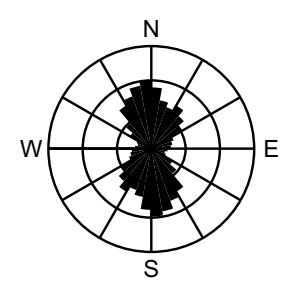

SW Off Hokkaido $(p=94 \%, N=1903)$

(d) Observed

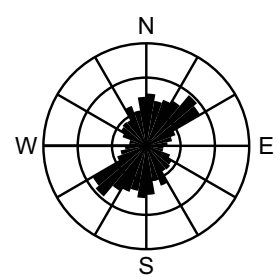

(e) Synthesized

(f) O/S Ratio

Miyake Island $(p=2.0 \%, N=1959)$

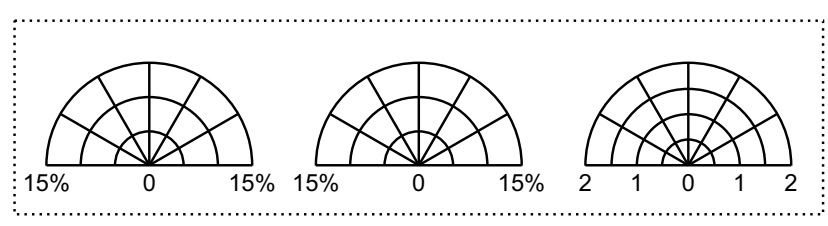

Fig. 3. Examples of the azimuthal distributions of the tidal compressional stress for the observed earthquake data (a and d) and for the synthesized random occurrence ( $b$ and $e$ ), and those of the $\mathrm{O} / \mathrm{S}$ ratio ( $c$ and $\mathrm{f}$; see text for definition). The distributions are plotted in rose diagrams, in which azimuthal angles are gathered into bins of $10^{\circ}$-width. Top figures are for the Southwestern Off Hokkaido subregion, and middle figures for the Miyake Island subregion, of which locations are shown in Fig. 2(a). The scales are shown at the bottom. distributions, we take the ratio of the proportion for the observed and synthesized data for each azimuth bin of $10^{\circ}$ width (Figs. 3(c) and 3(f)). We refer to this as "O/S ratio" hereafter. The $\mathrm{O} / \mathrm{S}$ ratio would take a value of about 1.0 in all directions in the case of no correlation between the Earth tide and earthquake occurrence, while it may deviate from 1.0 when there is a significant correlation. A high value of the $\mathrm{O} / \mathrm{S}$ ratio means that the corresponding azimuth is preferred by earthquake occurrence. For the Southwestern Off Hokkaido subregion (Fig. 3(c)), the O/S ratio distributes uniformly around 1.0 , which is consistent with the result of the statistical test. On the other hand, a high value of the $\mathrm{O} / \mathrm{S}$ ratio concentrates in the NW-SE direction for the Miyake Island subregion (Fig. 3(f)), where a significant correlation was confirmed by the statistical test. This indicates that earthquakes are encouraged to occur when the tidal compressional stress is in this direction in the Miyake Island subregion.

By introducing the $\mathrm{O} / \mathrm{S}$ ratio, we were able to extract the tidal compressional azimuth preferred by earthquake occurrence. Tanaka et al. (2002b) shows that earthquakes tend to occur when the tidal stress adds to the tectonic stress. From this result, it is expected that the anomalous azimuth may be aligned with the tectonic stress field. In Fig. 4, the azimuthal distribution of the $\mathrm{O} / \mathrm{S}$ ratio is compared with the frequency distribution of the $\mathrm{P}$-axis direction of focal mechanisms obtained in the corresponding subregion by JMA. The comparison is made for the ten subregions with $p<10 \%$ for which focal mechanism solutions are available. For all the subregions in this figure, a high value of the $\mathrm{O} / \mathrm{S}$ ratio is found to concentrate in and around the dominant direction of P-axes. In the case of Miyake Island subregion (Fig. 4(j)), for example, both the $\mathrm{O} / \mathrm{S}$ ratio and the frequency of Paxes take a high value in the NW-SE direction. This suggests that a small stress change due to the Earth tide may encourage earthquake occurrence when it acts in the direction to increase the regional tectonic stress.

In order to statistically compare the azimuthal distribution of the $\mathrm{O} / \mathrm{S}$ ratio with that of the $\mathrm{P}$-axis direction, we perform the $\chi^{2}$ test. In this test, we assess the significance level $\alpha$ to reject the null hypothesis that these two distributions are samples from the same population. For the ten subregions shown in Fig. 4, we obtain $\alpha=35-100 \%$. Large $\alpha$-values of $100 \%$ and $92 \%$ are obtained for the Aki Nada subregion of $p=8.6 \%$ (Fig. 4(c)) and the Miyake Island subregion of $p=2.0 \%$ (Fig. 4(j)), respectively, although not enough large $(\alpha=53 \%)$ for the Off Miyagi subregion of $p=6.8 \%$ (Fig. 4(h)).

\section{Discussion}

We attempt to make a rough estimate of the proportion of tidally triggered earthquakes. For this purpose, we sum up the excess numbers of the earthquakes over the numbers expected from the random earthquake occurrence for the directions with the $\mathrm{O} / \mathrm{S}$ ratio greater than 1.0. Taking the ratio to the total number of the earthquakes, we obtain $4.9-$ $11 \%$ for the subregions with $p<10 \%$. This suggests that a significant number of earthquakes may be triggered by the Earth tide.

Recent studies have indicated that the tidal effect on earth- 

O/S Ratio P-axis
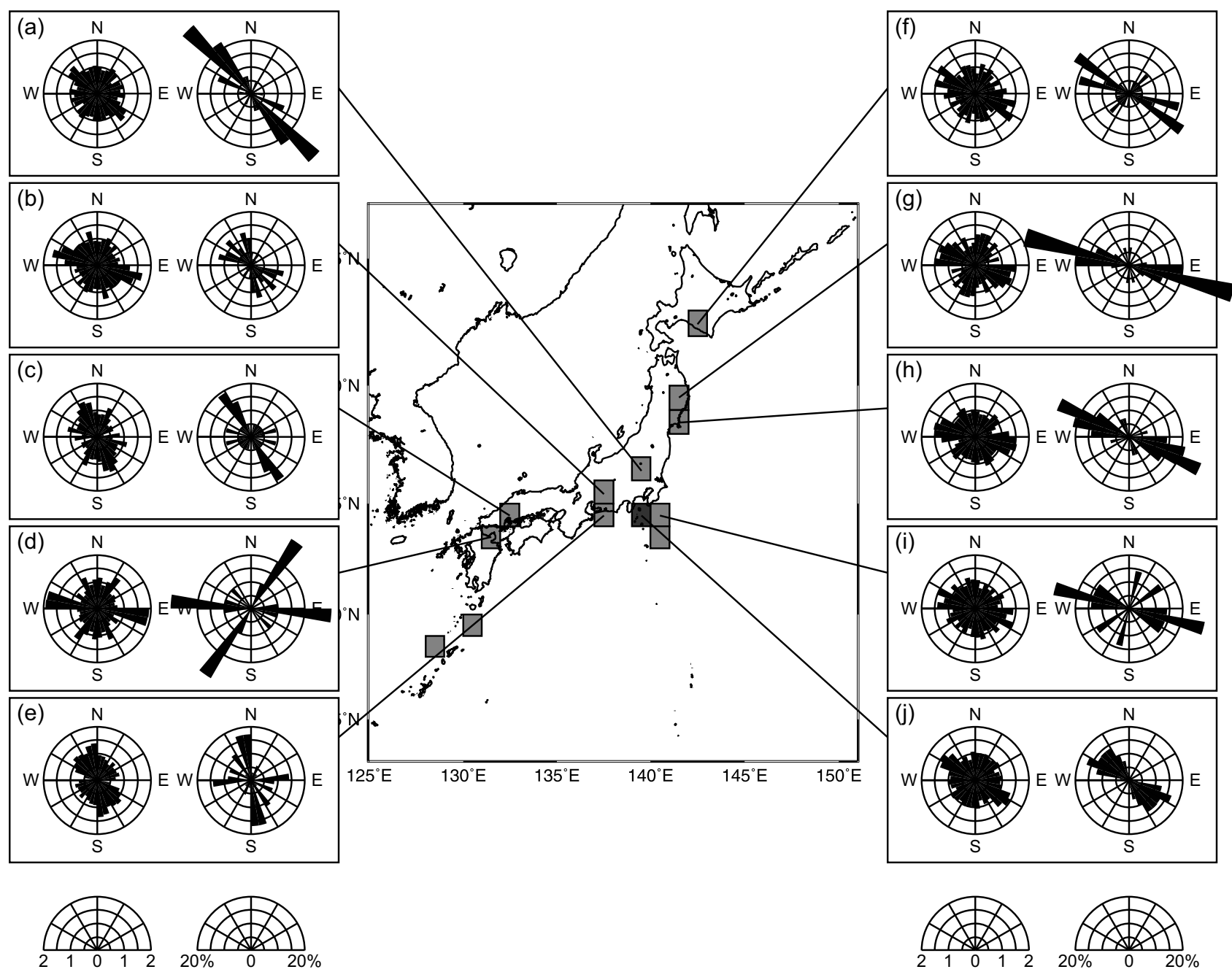

Fig. 4. Comparison between the azimuthal distribution of the $\mathrm{O} / \mathrm{S}$ ratio (left) and the $\mathrm{P}$-axis direction of focal mechanisms (right) for the ten subregions of $p<10 \%$ for which focal mechanism solutions are available. The scales are shown at the bottom.

quake occurrence may appear only when the stress state is approaching a critical level just before the occurrence of a large earthquake. Yin et al. $(1995,2000)$ proposed that the temporal change in seismic response to the tidal stress may be used for warning about an impending large earthquake and showed some successful cases. Tanaka et al. (2002a) showed that the high correlation between the Earth tide and earthquake occurrence was concentrated in and around the future focal region for the several years preceding the occurrence of the South Tonga earthquake $\left(M_{w} 7.5\right)$. In the light of these results, one interpretation of our results is that regions with a significant correlation may have a near-critical stress state. Indeed, in the Miyake Island subregion where the smallest $p$-value of $2.0 \%$ was observed, unusual seismic activity including five $M_{\mathrm{JMA}} \geq 6.0$ earthquakes took place in June-August 2000. Additionally, in the Aki Nada subregion of $p=8.6 \%$ (corresponding to Fig. 4(c)), the Geiyo earthquake ( $M_{\mathrm{JMA}} 6.7$ ) occurred on 24 March 2001. More recently, the Off Miyagi earthquake on 26 May 2003 ( $M_{\text {JMA }}$ 7.0) and the Northern Miyagi earthquake on 26 July 2003 $\left(M_{\text {JMA }}\right.$ 6.2) occurred in the Off Miyagi subregions, which correspond to Figs. 4(g) $(p=7.3 \%)$ and 4(h) $(p=6.8 \%)$, respectively. The positive correlation found in these subregions may be related to the occurrence of these seismic activities. On the other hand, a large $p$-value was obtained for the Southwestern Off Hokkaido subregion, where a large earthquake of $M_{\text {JMA }} 7.8$ occurred on 12 July 1993 preceding the investigation period. This is consistent with the result of Tanaka et al. (2002a) that the high correlation disappeared after the occurrence of the large South Tonga earthquake.

It is of particular interest to investigate the temporal change in $p$-value for the cases associated with large earthquakes. For the Miyake Island subregion, we perform the analysis dividing the earthquake data into two periods: before and after the seismic activity in June-August 2000. As a result of the analysis, a smaller $p$-value of $1.2 \%$ is obtained for the pre-seismic period, while a larger value of $17 \%$ for the post-seismic period. This is in good harmony with the result of Tanaka et al. (2002a). For the other subregions, the number of earthquakes is not enough at present to fully perform the analysis. The study will be done in the near future by adding newest data.

We note that the Off Tokachi earthquake on 26 September 2003 ( $M_{\text {JMA }}$ 8.0) occurred in an insignificant subregion (see 
Fig. 2(a)) but close to the significant subregion of $p=8.4 \%$ corresponding to Fig. 4(f). We also note that small $p$-values were observed for two subregions where the possibility of large earthquakes has been argued; the Tokai subregion (e.g., Ishibashi, 1981) of $p=7.8 \%$, and the Off Miyagi subregion (e.g., Ohtake and Ueda, 2002) of $p=6.8 \%$, which correspond to Figs. 4(e) and 4(h), respectively.

Earthquake triggering has been widely reported, especially on a good correlation of the Coulomb stress change produced by large earthquakes with the distribution of aftershocks (e.g., Harris, 1998; Stein, 1999). For example, an investigation for the 1992 Landers earthquake $\left(M_{w} 7.3\right)$ revealed that aftershocks were triggered by the stress increase greater than $10^{4} \mathrm{~Pa}$ (King et al., 1994). Most of these studies rely on the assumption that aftershocks occur on planes optimally oriented for failure, which are mainly dominated by the regional tectonic stress. Thus these observations are consistent with our finding that the earthquake occurrence was promoted by a small stress change to increase the regional stress. On the other hand, the amplitude of the tidal stress is only about one-tenth of the threshold for detection of the static triggering. Our observation provides new evidence that even such a small stress change can trigger an earthquake.

\section{Conclusion}

We examined the direction of the maximum principal axis of the tidal stress tensor at the origin times and the hypocenters of 89,504 shallow earthquakes occurring in the 100 subregions in Japan. The azimuthal distribution of the tidal compressional stress obtained for the observed earthquake data was statistically compared with that synthesized for random earthquake occurrence. Significant difference between the two distributions $(p<10 \%)$ was found for 13 subregions. For these subregions, we took the ratio of the proportion for the observed data to that for the synthesized random occurrence. High value of this ratio, which means that the corresponding azimuth is preferred by earthquake occurrence, was found to concentrate in and around the dominant direction of the $\mathrm{P}$-axes of focal mechanisms obtained in the corresponding subregion. This indicates that a small stress change due to the Earth tide can trigger an earthquake when it acts in the direction to increase the regional tectonic stress, in consistent with recent observations on seismicity change associated with the occurrence of large earthquakes. Among possible factors of earthquake triggering, the Earth tide is of particular interest since it exists universally, and is theoretically predicted with sufficient accuracy. Studies on tidal triggering of earthquakes may provide a new clue to reveal the physical mechanism of rupture initiation.

Acknowledgments. We are grateful to W. Zurn and R. S. Stein for their helpful comments to the manuscript. We used the earthquake catalog arranged by the Japan Meteorological Agency in cooperation with the Ministry of Education, Culture, Sports, Science and Technology. This study was partially supported by Grant-in-Aid for JSPS Fellows (No. 07136).

\section{References}

Dziewonski, A. M. and D. L. Anderson, Preliminary reference Earth model, Phys. Earth Planet. Inter., 25, 297-356, 1981.

Emter, D., Tidal triggering of earthquakes and volcanic events, in Tidal Phenomena, Lect. Notes Earth Sci., vol. 66, edited by H. Wilhelm, W. Zurn, and H.-G. Wenzel, pp. 293-309, Springer-Verlag, Berlin, 1997.

Glasby, G. P. and J. Kasahara, Influence of tidal effects on the periodicity of earthquake activity in diverse geological settings with particular emphasis on submarine by hydrothermal systems, Earth Sci. Rev., 52, 261-297, 2001.

Harris, R. A., Introduction to special section: Stress triggers, stress shadows, and implications for seismic hazard, J. Geophys. Res., 103, 24,34724,358, 1998.

Ishibashi, K., Specification of a soon-to-occur seismic faulting in the Tokai district, central Japan, based upon seismotectonics, in Earthquake Prediction: An International Review, edited by D. W. Simpson and P. G. Richards, pp. 297-332, American Geophysical Union, Washington D. C., 1981.

King, G. C. P., R. S. Stein, and J. Lin, Static stress changes and the triggering of earthquakes, Bull. Seismol. Soc. Am., 84, 935-953, 1994

Matsumoto, K., T. Takanezawa, and M. Ooe, Ocean tide models developed by assimilating TOPEX/POSEIDON altimeter data into hydrodynamical model: A global model and a regional model around Japan, J. Oceanogr., 56, 567-581, 2000.

Ohtake, M. and H. Ueda, Statistical forecast of the next Miyagi-Oki, northeast Honshu earthquake, Tohoku Geophys. J., 36, 299-310, 2002.

Reasenberg, P., Second-order moment of central California seismicity, 1969-1982, J. Geophys. Res., 90, 5479-5495, 1985.

Stein, R. S., The role of stress transfer in earthquake occurrence, Nature, 402, 605-609, 1999.

Takanezawa, T., K. Matsumoto, M. Ooe, and I. Naito, Effects of the longperiod ocean tides on Earth rotation, gravity and crustal deformation predicted by global barotropic model-periods from $\mathrm{Mtm}$ to $\mathrm{Sa}-, J$. Geod. Soc. Japan, 47, 545-550, 2001.

Tanaka, S., M. Ohtake, and H. Sato, Spatio-temporal variation of the tidal triggering effect on earthquake occurrence associated with the 1982 South Tonga earthquake of Mw 7.5, Geophys. Res. Lett., 29(16), 1756, doi:10.1029/2002GL015386, 2002a.

Tanaka, S., M. Ohtake, and H. Sato, Evidence for tidal triggering of earthquakes as revealed from statistical analysis of global data, J. Geophys. Res., 107(B10), 2211, doi:10.1029/2001JB001577, 2002b.

Tsuruoka, H., M. Ohtake, and H. Sato, Statistical test of the tidal triggering of earthquakes: Contribution of the ocean tide loading effect, Geophys. J. Int., 122, 183-194, 1995.

Vidale, J. E., D. C. Agnew, M. J. S. Johnston, and D. H. Oppenheimer, Absence of earthquake correlation with Earth tides: An indication of high preseismic fault stress rate, J. Geophys. Res., 103, 24,567-24,572, 1998.

Wilcock, W. S. D., Tidal triggering of microearthquakes on the Juan de Fuca Ridge, Geophys. Res. Lett., 28, 3999-4002, 2001.

Yin, X. C., X. Z. Chen, Z. P. Song, and C. Yin, A new approach to earthquake prediction: The load/unload response ratio (LURR), Pure Appl. Geophys., 145, 701-715, 1995.

Yin, X. C., Y. C. Wang, K. Y. Peng, Y. L. Bai, H. T. Wang, and X. F. Yin, Development of a new approach to earthquake prediction: Load/unload response ratio (LURR) theory, Pure Appl. Geophys., 157, 2365-2383, 2000.

S. Tanaka (e-mail: tanaka@bosai.go.jp), M. Ohtake, and H. Sato 